\title{
Journal of Research in Nursing
}

http://jrn.sagepub.com

\section{Preparing the air for nursing care: A grounded theory study of first line nurse managers \\ Terese Bondas}

Journal of Research in Nursing 2009; 14; 351

DOI: $10.1177 / 1744987108096969$

The online version of this article can be found at:

http://jrn.sagepub.com/cgi/content/abstract/14/4/351

\author{
Published by: \\ @SAGE \\ http://www.sagepublications.com
}

Additional services and information for Journal of Research in Nursing can be found at:

Email Alerts: http://jrn.sagepub.com/cgi/alerts

Subscriptions: http://jrn.sagepub.com/subscriptions

Reprints: http://www.sagepub.com/journalsReprints.nav

Permissions: http://www.sagepub.co.uk/journalsPermissions.nav

Citations http://jrn.sagepub.com/cgi/content/refs/14/4/351 


\title{
Preparing the air for nursing care: A grounded theory study of first line nurse managers
}

\section{Terese Bondas}

Journal of Research

in Nursing (C)2009

SAGE PUBLICATIONS

Los Angeles, London,

New Delhi and Singapore

VOL 14 (4) 351-362

DOI: $10.1177 /$

1744987108096969

\section{Professor}

University of Borås, Sweden Associate Professor, Kuopio University, Finland

Chair of BFiN and ChuCE

\begin{abstract}
The first line nurse managers' opportunities to lead nursing care seem to be diminishing. The aim of this study was, therefore, to gain an understanding of the first line nurse managers in their experiences in the development of nursing care as part of a wider research programme. Finnish nurse managers wrote narratives at the beginning of five different leadership courses in this grounded theory study.

'Preparing the Air for Nursing Care' emerged as a core category. It was formed by two major categories. 'Being Concerned about Nursing Care' describes the nurse managers' focus on the development of nursing care, the nursing caregivers' health and knowledge and a concern for the whole organisation. The second major category 'Creating the Direction and Content of Nursing Care' describes the nurse manager working together with the staff to create individual and family-centred best practice, initiating relationships and dialogues for nursing care, and a culture of caring. A typology was created that explained the four main modalities to emerge from the data: 'the Active Developer', 'the Passive Thinker', 'the Impulsive Creator' and 'the Routine Manager'.
\end{abstract}

Key words first-line nurse manager; grounded theory; nursing care development; nursing leadership; nursing management

\section{Introduction}

The first line nurse managers' opportunities to lead nursing care seem to be diminishing in complex health care organisations. Research into evidence-based nursing has pointed to the importance of nursing leadership. Nursing leadership and management in the development of nursing care seem to be related to complex phenomena in the organisational culture of the unit as well as phenomena related to the nurse managers and their background and interests. There is research into overall characteristics of nursing leadership. However, to develop evidence-based care, more understanding is needed about the meaning of nursing leadership in the development of care from the nurse managers' perspective. The aim of this study was, therefore, to 
gain an understanding from the perspective of the first line nurse managers themselves, and their experiences in the development of nursing care as part of a wider research programme into nursing leadership.

\section{Background}

Clinical leadership and nursing care knowledge may be considered as an ambiguous part of nursing management (Antrobus and Kitson, 1999; Narinen and Kekki, 2003; Upenieks, 2003; Stanley, 2006a,b). The first-line nurse manager may handle the ambivalence and insecurity by being one of the nurses who adds administrative tasks to the nurse profile (Johansson, et al., 2007), or on the contrary, nurse managers may distance themselves from the every day world of nursing care, primarily working to their own agendas (Stanley, 2006a,b). Sellgren, et al. (2006) found dominant profiles of an invisible nurse manager and a 'middle of the road' manager in nursing care. Nurse managers often obtain their positions by chance or as a temporary solution rather than choosing their nursing careers in a spirit of ideals, and a conscious nursing career choice (Bondas, 2006). It may also be difficult to maintain the human values of ethical nursing care in the turbulent environment of the health care organisations. Visions of nursing care may easily get lost in the turbulence of the daily administrative matters and routines if the visions are not sustained (Graham, 2003). Vision was hardly mentioned as an aspect of clinical leadership in a British study. However, it was more important that the clinical leaders lived their passion about nursing care (Stanley, 2006a,b).

Advanced knowledge of nursing science and health care administration in health care organisations is not always appreciated (McKenna, et al., 2004) and the worth of management training was hardly recognised by the clinical leaders themselves (Stanley, 2006a). There is a lack of appropriate educational qualifications in research and science to develop nursing care, leadership, administration and health economics (Duffield and Franks, 2001). Confusion and disagreement have been expressed about the survival of nursing leadership (McKenna, et al., 2004), whereas administrative positions have been occupied by non-nurses who lack knowledge in nursing care (Mahoney, 2001). The first line nurse managers' opportunities to lead nursing care together with the nursing professionals seem to be diminishing in the new public management direction of health nursing care. The ideas and practice of nursing leadership and management based on human dignity, human love and mercy have also been set aside by introducing management practices from industry and business sectors (Bondas, 2003).

Clinical nursing leadership is considered an under-researched phenomenon from a nursing science perspective, and much of the literature has been studied from business, general administration or industrial perspectives (Davidson, et al., 2006) There have been many studies from other perspectives such as attributes, traits, qualities, competencies, roles and styles focusing on what the nurse leaders do in their work and who they are (Mahoney, 2001; Drach-Zahavy and Dagan, 2002; Wieck, et al., 2002; Narinen and Kekki, 2003; Upenieks, 2003; Carroll, 2005; Sellgren, et al., 2006). A recent systematic review shows that the nurse managers need to have an understanding of the key factors associated with producing a positive climate in the organisation and to undertake development activities in nursing care (Pearson, et al., 2007).

Nursing leadership is also regarded as the cornerstone of evidence-based practice programs within health care organisations (Driever, 2002; Banning, 2005; Gifford, 
et al., 2007). Nursing leaders in any management position combined their sphere of influence with practicing nursing, and they valued nursing knowledge (Antrobus and Kitson, 1999; Lindholm, et al., 2000; Boykin and Schoenhofer, 2001; Drach-Zahavy and Dagan, 2002; Narinen and Kekki, 2003; Upenieks, 2003). Viitanen, et al. (2007) found the role of the nurturing mother, who is committed to take care of the staff. However, it seems to be difficult for the nurse leaders to understand the differences between providing bedside nursing care and clinical nursing leadership. Thus, there is research in nursing leadership from a leader-centred competence, role or profileperspective where nursing care is an ambiguous part beside leadership and management and findings from research in evidence-based care that highlights the importance of nursing leadership. It is thus important to study how first line nurse managers themselves experience their clinical nursing leadership in relation to nursing care development.

\section{Aim}

The aim of this study was to gain an understanding of the first line nurse managers' experiences of the development of nursing care as part of a wider research programme into nursing leadership.

\section{Method and participants}

A qualitative method that applies comparisons and contrasting procedures and theory generation according to the grounded theory tradition was chosen (Glaser and Strauss, 1967; Glaser, 1978). The data were collected by narratives. The questions for the narratives were based on the previous findings of the research programme and a review of the literature. The open questions used were: 'How would you describe your everyday leadership practice in relation to nursing care?', 'How would you describe the core of your nursing leadership?' and 'What do you want to develop as a nurse manager in your organisation?' The proposal was to allow the nurse managers freely to tell their story, describe their experiences and reflect on them. Background data about age, position, years of working experience and education were also collected.

Sixty-five first line nurse managers (60 women and 5 men) took part in this qualitative study. Variation of age, education and experience of nursing and nursing leadership characterised the sample. The participants were employed as ward managers in both rural and urban areas; in small health nursing care centres as well as in hospitals of various sizes and specialties. Their educational backgrounds varied except for their nursing degrees, and they were all specialised in a certain area of nursing care. Only a few of the informants (5\%) had completed master's programs, whereas $71 \%$ had participated in various courses in leadership and administration, which had not led to a degree. Additionally, $24 \%$ of the participating nurse managers did not report any administrative education apart from their nursing education. The participants' experience as managers ranged between 3 months and 26 years. All were experienced Registered Nurses. Their age ranged between 25 and 55 years. The units where they were employed had between 4 and 30 staff positions.

The settings of the study were different leadership courses, and the participants were asked to write narratives based on the three open questions at the beginning of their course. The courses in which the participants were enrolled varied. They were either short mandatory or voluntary in-service courses for nurse managers, or 
leadership courses which were part of a master's degree. Data collection continued in every new course until saturation was reached in the analysis, that is, no new codes emerged from the data (Glaser and Strauss, 1967). Narrative descriptions ranged from a few lines to detailed descriptions and four pages stories.

\section{Data analysis}

The data were read, reread and analysed line by line after the data collection at every course, and finally read again, as a whole, when no new codes emerged. The researcher was open to the variation in the data searching for codes and contradictions in the data. Categories were identified after coding. Each text line was read and analysed again and again, going back and forth from the data to the analysis and between the participants, comparing and contrasting the findings. A core category is proposed that is grounded in the data, and it is constructed from the relationships among the main two categories. The combination of the main categories created the basis for a proposed typology (Glaser and Strauss, 1967; Glaser, 1978). Four qualitatively different types of nursing leadership in relation to nursing care development emerged from the analysis.

\section{Ethical considerations}

The study was of part of a departmental research programme into nursing leadership. Each participant was given an information sheet in addition to a verbal explanation and a possibility to ask questions. They were informed that it was voluntary to participate and they could withdraw from the study at any time. The anonymity of the participants was protected. The participants were later informed of the preliminary findings and were able to discuss them with the researcher.

\section{Results}

\section{Preparing the air for nursing care}

The core category, 'Preparing the Air for Nursing Care', was discovered and explained through two major categories: 'Creating the Direction and Content of Nursing Care' and 'Concerned about Nursing Care'. The construction 'Preparing' is worded as a process and it means making possible the best available nursing care at the unit. The preparation in nursing leadership emerged as a continuing and neverending process for the nurse managers. 'Preparing' is important for the core function of the unit; i.e. to create the direction of nursing care and focus on the outcomes of care. 'Preparing' is a meaningful act when it connects to the main function; the care of the patients. The proposed core category that emerged in this study may guide and provide the focus for nursing leadership. The 'Air' as a metaphor also implies a continuous movement. The breathing in and out brings nourishment and a vital exchange of news, knowledge and relations for the unit and its patients, relatives and staff. In order to keep the 'Air' fresh, the unit needs doors and windows that are always open to the surrounding environment. A closed unit does not breathe; it can wither and die without creating new life. The core category was also expressed in these words by some of the participants. 


\section{Creating the direction and content of nursing care}

The first main category is called 'Creating the Direction and Content of Nursing Care'. Nurse managers described their role in nursing care as providing knowledge, models and ideals, directly or indirectly, through discussions to guide the nursing care at their unit. Differences in opinion of nursing care existed among the staff. However, differences in opinion leading to conflicts were mentioned often in relation to the co-operation with the physicians or staff at other units. The nurse leaders wanted to create the best individual nursing care practice, and the family was seen as important for the patient's wellbeing, but in its uniqueness, difficult to incorporate in nursing care. Relationships and dialogues built the basic ground for nursing care and formed the direction of nursing care at their unit, according to the nurse leaders. The nurse leaders viewed the culture of nursing care as an important expression of the specific ways of providing nursing care and the preferred caring practices at their unit. Three categories emerged: 'Creating Individual and Family-centred Nursing Care', 'Creating Relationships and Dialogues for Nursing Care' and 'Creating a Culture for Nursing Care’.

\section{Creating individual and family-centred nursing care}

The nurse managers described the patients as important guides for their leadership. The ideal that they strove for was nursing care on their unit that was based on knowing each patient: 'It is our intention to make the patients feel that we are there for them and offer them the time they need, and they are satisfied with the nursing care when they leave us'. The nurse managers described that the worries of the patients and their relatives were taken seriously. Nurse managers recognised ethical dilemmas in nursing care, and they knew that their staff needed support and help to deal with the daily prioritisation in caring for their patients. They were also concerned with shortcomings in nursing care, omitted nursing care and the staff's ignorant behaviour. A nurse manager described her intention: 'I want to be the patients' advocate and never accept violations of their dignity, and become more courageous in taking a stand when I see unnecessary suffering'.

The participants who had a master's degree or were master's students described nursing science as a basis for nursing care and their leadership. They also emphasised the meaning of listening to the patients, a practice which the participants recognised as important for the development of nursing care. A nurse manager called her participation in the daily nursing care of the patients 'an inside quality assurance'. Pain alleviation was mentioned as an important matter to develop. The nurse managers' main responsibility was, however, being in charge of providing nursing care that maintains and furthers the patient's health and daily living. They did not want their staff to regard them as controlling or experts who always knew better than the staff. Nurse managers also described their difficult position in nursing care when they tried to help their staff. They wanted to show respect for their staff's knowledge and not get too involved in issues that they felt that their staff knew best.

\section{Creating relationships and dialogues for nursing care}

The nurse managers considered the development of collaborative relationships and ongoing dialogues with the patients, their relatives, staff and different stakeholders as pivotal to their role. It was considered important to receive and give feedback regarding nursing care practices. Hierarchy seemed to persist in the nurse managers' descriptions as a difficult challenge to overcome and sometimes accept. They felt a 
need to stand up for their unit and their staff when they perceived threats against their existence or unjust complaints about their practice. The nurse managers described that they, sometimes, had to fight for the ideas that they believed in. They felt that it was a balancing act because they did not want to hurt anybody. Nurse managers also dealt with problem solving and every day conflicts, and emphasised the importance of listening, supporting, motivating and understanding the staff's views and their different perspectives: 'It is important to create safety (support and knowledge) for the staff, so that they will walk an extra mile for their patients'. Nurse managers described closeness to the staff that provided possibilities to monitor what was going on and notice improvement needs: 'Seeing the human being and listening to the patients and the staff at my unit'.

The nurse managers also described their own need to be seen as vulnerable human beings. A first line nurse manager described conflicts at her unit and the fact that she 'got plenty of support from the nurse executive during that difficult period'. It felt bitter to be stabbed in the back and she did not know what was going on at the unit. There were also descriptions of nurse managers' personal change from being authoritative and controlling in relationships with the staff at younger age to become more flexible, generous, spontaneous, secure and positive with staff of increasing age and experience. Assertiveness and patience were desired traits for the participating nurse leaders in relation to their staff.

\section{Creating a culture for nursing care}

The nurse managers felt responsible for how people experienced the organisational culture of their unit. They hoped that they could create an atmosphere of caring based on ethical values of humanity. Human dignity was mentioned as an imperative, which required constant attentiveness. The nurse managers described how their value base was created and clarified through reflections and discussions about problematic situations in nursing care as well as through handling conflicts at the unit. The value base was metaphorically compared with the 'main thread that is woven to a fabric with beautiful patterns' or 'glue that holds the staff together'. The nurse managers emphasised the creation of an esthetical environment, which was regarded as important for the sense of wellbeing of both patients and staff. Functional aspects of the environment, such as the size of rooms, were also mentioned. It was seen as important to create a good spirit at the unit, including humour. The culture was described by cornerstones, such as trust, safety, supporting contacts, flexibility and openness. An innovative climate was desirable, and the experienced nurse leaders recognised that it was seldom possible to wait for the optimal time for development. According to the nurse managers, there were always some problems or a rush. The nurse managers described that they had to catch the moment; 'to evaluate and openly examine what has been going on and learn' and actively 'stir and initiate a movement if needed'. The nurse managers wanted to discuss and reflect on different matters and situations in nursing care with the staff. According to the nurse managers, the only reason for change should be related to the patients and their needs: 'Listening to the patient's experiences and using my understanding in changing my unit towards a more caring culture'. One of the nurse managers used 'air' as a metaphor for the life-giving oxygen to the unit: 'My task as a nurse manager is to prepare the air to motivate the staff to create good nursing care and to develop nursing care'. The organisation could be described in terms of ill health. Change processes could take a long time, which created boredom. Some nurse managers experienced the wished-for 
cultural change as a hopeless and endless process, which made them think about giving up the change processes or leaving.

\section{Being concerned about nursing care}

The second major category is called 'Being Concerned about Nursing Care'. Nurse managers described that they often had nursing care on their mind, and they expressed their ideals for the development of nursing care. They recognised the importance of the nursing caregivers' will and knowledge of nursing care, and described that they were lucky to have a knowing and caring staff. The importance of the health care centre or hospital as a human centred organisation was emphasised. The sub-categories are: 'Concern for the Development of Nursing Care', 'Concern for the Staff' and 'Concern for the Organisation'.

\section{Concern for the development of nursing care}

The nurse managers were concerned with the direction, organisation and coordination of nursing care: 'We all work for the best of the patient; it is the core idea in every aspect of our work'. They regarded professional cooperation in nursing care as a very important issue and therefore preferred an open view of knowledge. The nurse managers considered themselves responsible for the continuous movement and development at their unit and the search for knowledge and innovations. However, it took time to find a satisfying and meaningful position. The shift from the nurse's responsibilities for the individual patient's nursing care to the nurse manager's responsibilities for nursing care development at the whole unit was described as challenging and difficult. The nurse managers described that they paid attention to nursing care of both human and material resources. One nurse manager pointed out: 'each staff member is considered important and everyone's ideas of development should be honoured'. The nurse managers wanted to involve everyone in the best nursing care and safeguarded specialised knowledge in nursing care. Responsibility for nursing care was also connected to nursing research, staying ahead of new innovations and new technology.

\section{Concern for the staff}

The nurse managers described that they wanted to support their staff. They also expected the best performance of their staff. An ideal that the nurse manager strove towards was supporting knowing, engaged and caring staff members who would easily understand the importance of supporting patients and their relatives. The nurse managers perceived that it was their task to recruit and maintain staff. They tried to involve the staff in planning and evaluation, in the formulation of the ideals and visions of nursing care, and in the effort to achieve the common goals. They tried to be just in personnel politics and related their decisions to the guiding ideas of nursing and caring. Being fair was described as a difficult task: 'I try to treat everyone in a fair way within reasonable limits; it is rather ungrateful, you seldom get thanks but immediate critiques when wishes cannot be fulfilled'. The nurse managers described how they cared for nursing staff: 'If the staff does not feel well, then the whole organisation suffers too'. An atmosphere of hopefulness and joy at work is described to have positive effects on the patients and their families at the units. Time for reflections on evidence-based nursing care and innovations was seen as important. There were, however, scarce descriptions of the nurse managers' actual practice. The responsibility issue was often mentioned by the nurse managers: 'It is 
sometimes difficult when nursing caregivers do not take responsibility; they only do their work if there is someone to tell them what to do and how to do it. They just cannot see the patients' needs. Freedom may also be a threat to an insecure person who wants me to be the manager that knows and takes care of everything'. The nurse managers recognised the importance of continuous education for all staff members and they described planning for continuous staff education and their nursing careers. The nurse managers' own education was not often mentioned, though.

\section{Concern for the organisation}

The participating nurse managers described that they wanted to be proud of their organisation, and they felt responsible for the health of the whole organisation in which they were employed. These feelings had changed and developed during their management nursing careers: 'It is not just about being there, but truly choosing this kind of work and this organisation'. There were also descriptions of participating in important work for human beings, love for life and human beings. According to the nurse managers, the difference between their former nursing career and nursing leadership is liability. When they were nurses, they took responsibility for patients in their nursing care and the tasks they were assigned to. As managers, they felt responsible for their unit, some mentioned responsibility for the whole organisation and the use of health nursing care resources. It is important to collaborate 'in all directions' as well as 'to spread knowledge about the unit and the nursing care that we provide'. The nurse managers looked upon themselves as experts when it came to nursing care in their unit, which meant that the world outside their organisation was not that important. A problem for the nurse managers was their attitude to the daily routine matters and problems, which always seem to pop up and block planning, vision, development work and major organisational matters. The nurse managers recognised that these matters would not appear as easily if they had enough time and knowledge available to engage in issues other than managing routine matters and practicing immediate problem-solving. The struggle for material resources was not evident in the answers. It seemed that the nurse managers were content to accept the limitations and their situation as it was. 'I try to create a world of nursing care in our organisation, as good as possible, based on the economic resources that have been provided'. It was also important to obey the laws and see that there was enough knowledge at their unit about the legal framework of health nursing care.

\section{Different modalities of the core category}

Different modalities of the main category 'Preparing the Air for Nursing Care' emerged from the variations of the relationship between the two major categories 'Creating the Direction and Content of Nursing Care' and 'Being Concerned about Nursing Care'. This study of nursing leadership led to the development of a typology including four variations: the Active Developer, the Passive Thinker, The Impulsive Creator and finally the Routine Manager. The Active Developer combines both concern about nursing care and creation of nursing care in their leadership: 'Nursing science is a part of my nursing leadership and I act accordingly to the values and knowledge'. The Passive Thinker is dominated by good ideas and is concerned about nursing care, but there seem to be too little translation of their concerns as nurse managers to practice: 'There is a lack of time for development of new ideas; I get too involved in the nursing care'. The Impulsive Creator is characterised by an 
unplanned ad hoc creation of development, whereas concern may be missing: 'I feel insecure in many situations, too emotional and too much temperamental. I have had many ideas, but I have difficulties in delegation, and I do not give enough room for my staff, and then it is so easy for me to dominate'. Finally, the Routine Manager is mostly interested in accomplishing administrative tasks but he/she seems to lack concern for the content and direction of nursing care: "The time schedule for the staff take a lot of my time, meetings, answering the phone and then I try to do the paperwork well in advance'. The typology suggests that the core category 'Preparing the Air for Care' may be found in four modalities.

\section{Discussion}

The core category of nursing leadership in relation to the development of care emerged and was described as Preparing the Air for Nursing care. It is explained through two major categories: 'Being concerned about Nursing Care' and 'Creating the Direction and Content of Nursing Care'. A typology has also been offered as a result of the analysis and the modalities are: the Active Developer, The Passive Thinker, the Impulsive Creator and the Routine Manager. These findings add and deepen the insights into nursing leadership in relation to nursing care and support other studies concerned with the nurse manager's different attributes, roles and skills (Mahoney, 2001; Drach-Zahavy and Dagan, 2002; Wieck, et al., 2002; Narinen and Kekki, 2003; Upenieks, 2003; Carroll, 2005; Sellgren, et al., 2006). This study was, however, confined to nurse managers at the ward level in Finland. Nevertheless, the findings may be transferred to other countries with similar nursing care and nursing leadership positions. The categories were discovered from the data and were not pre-selected, and the data analysis was done according to grounded theory approach (Glaser and Strauss, 1967; Glaser, 1978). Saturation in data analysis was safeguarded through a recurring data collection and analysis with comparing and contrasting line by line analysis until no new codes emerged. A core category emerged from the data analysis and a typology was constructed based on the two major categories explaining the variations. The findings of the preliminary analysis were reported to the participants for fit, and there were expressions of recognition that the findings were meaningful. The findings also started lively discussions in the groups and were regarded as relevant to their practice. The work criterion means that the findings provide explanations and interpretations of what is going on. It is possible to modify and develop a tentative theory (Glaser and Strauss, 1967; Glaser, 1978).

The nurse managers described that they tried to influence their unit and their world of nursing care, which could be compared with the classical views of leadership and power. The main characteristic of nursing leadership is, however, its unique purpose - providing the best possible nursing care. It signifies the special atmosphere, the sphere, the exchange and the movement that are needed for nursing care to take place (Stanley, 2006a,b). The metaphorical construct 'Preparing the Air for Nursing Care' adds knowledge to the caritative leadership theory, especially the meaning of nursing leadership in the creation of a caring culture and the relationships and dialogues in the interest of ministering to the patient (Bondas, 2003, 2006). The reciprocal collaborative relationships with the staff pop up as ideals, but paradoxically, they remain hidden in the fact that the nurse managers' receptivity and their own change and growth are not that visible in the findings. This might also signal the loneliness and insecurity that the nurse managers hide in trying to be proficient and perfect. The research of transformational leadership shows the risks of 
the ideal of the perfect manager and the blind eager followers who place the manager on a throne, which can lead to unfavourable outcomes, such as bad decision-making and dysfunctional organisations (Reinhardt, 2004).

Cultural change is also connected to the respect for the discipline of nursing and knowledge of an evidence-based profession (McKenna, et al., 2004; Gifford, et al., 2007). These types of cultural concerns create involvement in nursing care. However, these concerns are insignificant to a manager without knowledge and understanding of nursing care (Viitanen, et al., 2007) or visions of nursing care (Graham, 2003). In this study, an example is the Routine Manager typology, who accomplishes administrative tasks, but leaves the nursing care development to her/his staff without understanding their role.

Moreover, there is a strongly expressed concern for the staff, an idea that might be related to the nurse managers' nursing education and experience of patient nursing care. This finding is in line with the role of the nurturing mother in the focus group study of Viitanen, et al. (2007), the observation study of Drach-Zahavy and Dagan (2002) as well as the Congruent Leadership theory (Stanley, 2006a,b). However, the task of nursing leadership is also to prevent ill health because of the work conditions. The paradox of nursing leadership becomes evident; the focus is double in a special way. The primary focus is patient nursing care, which is provided by professional nursing caregivers and depends on their health, will, competence, strength, and the nurse manager's support. The nurse leader needs patience and understanding of the situation at their unit, and not to be running their own race and hurrying into an unplanned or unrealistic change as the Impulsive Creator in this study.

It is not enough to have knowledge of nursing and knowledge about nursing care; the nurse manager has to translate it into development practices. Nursing leadership has the potential of safeguarding an ethical culture of nursing care, where the staff are able to concentrate on nursing care. The nurse manager acts as the Active Creator in relation to the development of nursing care; and it is not enough to feel concerned about nursing care as the Passive Thinker typology in this study suggests. The findings are also in line with Upenieks' study on effective leadership in magnet and nonmagnet nurse managers (2003) and Stanley's study on clinical leadership (2006a,b). The literature on evidence-based nursing care and nursing also shows that leadership is the cornerstone in the change process (Driever, 2002; Banning, 2005; Gifford, et al., 2007).

Longitudinal cultural studies based on nursing leadership and its impact on nursing care and interventional studies on nursing leadership in relation to nursing science knowledge and outcomes of phenomena in the organisational culture are needed. How do the nurse managers solve the role conflicts and co-operate with stakeholders with different interests that may blur their passion for nursing care, and how may the visions be sustained in a long-term nursing care development process? The question of nursing leadership is important in outlining and maintaining the ethical value base, direction and content of nursing care in the complex demands of an evidence-based, efficient and cost-effective nursing care. In the light of the findings in this study, the nurse managers seem to have the will but not always the knowledge or courage to mould his or her identity towards a synthesis of a nursing care knowledge in combination with leadership and management knowledge. Another question concerns the health nursing care organisations; do they recognise the nurse manager's potential or is it too late to show their knowledge? 


\section{Conclusions}

The basic process of nursing leadership in relation to development of nursing care from the first line nurse managers' perspective is 'Preparing the Air for Nursing care'. It supports evidence-based nursing care with a starting-point in relationships and dialogues with patients, their relatives, nursing caregivers and the world outside the unit. The 'Air for Nursing care' is 'prepared' together with the staff, by the nurse manager's 'Concern about Nursing care' in combination with 'Creation of the Direction and Content of Nursing care'. The main typology of nursing leadership include the Active Developer, the Passive Thinker, the Impulsive Creator and the Routine Manager. There is a need for a continuous ethical reflection that appears from nursing care, leadership and administration. Nurse managers may show their concern and create nursing care to continuously raise the bar for their staff and create the best ethical caring culture in their unit(s) that serves the patient, their relatives and creates an effective working environment for the staff.

\section{Key points}

- Nursing leadership has been studied from various perspectives such as attributes, traits, qualities, competencies, roles and skills. There is scarce research on the first line nurse managers' own perspective on clinical nursing leadership and their experiences of nursing care development.

- 'Preparing the Air for Nursing Care' is suggested as the core category of this grounded theory study on the basis of the first line nurse managers' concern about nursing care and their creation of the direction and content of nursing care.

- A typology was identified for nursing leadership in relation to the development of nursing care: 'the Active Developer', 'the Passive Thinker', 'the Impulsive Creator' and 'the Routine Manager'.

\section{References}

Antrobus, S, Kitson, A (1999) Nursing leadership: influencing and shaping health policy and nursing practice. J Adv Nurs 29: 746-753.

Banning, M (2005) Conceptions of evidence, evidencebased medicine, evidence-based practice and their use in nursing: independent nurse prescibers' views. J Clin Nurs 14: 411-417.

Bondas, T (2003) Caritative leadership: Ministering to the patients. Nurs Adm Q 27: 249-255.

Bondas, T (2006) Paths to nursing leadership. J Nurs Manag 13: $1-8$.

Boykin, A, Schoenhofer, S (2001) The role of nursing leadership in creating caring environments in health nursing care delivery system. Nurs Adm Q 25: 1-7.

Carroll, TL (2005) Leadership skills and attributes of women and nurse executives. Challenges for the $21 \mathrm{st}$ century. Nurs Adm Q 29: 146-153.

Davidson, PM, Elliott, D, Daly, J (2006) Clinical leadership in contemporary clinical practice: implications for nursing in Australia. J Nurs Manag 14: 180-187.
Drach-Zahavy, A, Dagan, E (2002) From caring to managing: an examination of the head nurse's role. J Adv Nurs 38: 19-28.

Driever, MJ (2002) Are evidence-based practice and best practice the same. West J Nurs Res 24: 591-597.

Duffield, C, Franks, H (2001) The role and preparation of first-line managers in Australia: where are we going and how do we get there. J Nurs Manag 9: 87-91.

Gifford, W, Davies, B, Edwards, N, Griffin, P, Lybanon, V (2007) Managerial leadership for nurses' use of research evidence. Worldviews Evid Based Nurs 4: 126-145.

Glaser, B (1978) Theoretical Sensitivity. Mill Valley, CA: Sociology Press.

Glaser, B, Strauss, AL (1967) The Discovery of Grounded Theory. Chicago: Aldine Publishing company.

Graham, I (2003) Leading the development of nursing within a Nursing development unit: the perspectives of leadership by the team manager and a professor of nursing. Int J Nurs Pract 9: 213-222. 
Johansson, G, Pörn, I, Theorell, T, Gustafsson, B (2007) A first-line nurse managers' goal profile. J Clin Nurs 16: 149-159.

Lindholm, M, Sivberg, B, Udén, G (2000) Leadership styles of nurse managers in changing organizations. J Nurs Manag 8: 327-335.

Mahoney, J (2001) Leadership skills for the 21st century. J Nurs Manag 9: 269-271.

McKenna, H, Keeney, S, Bradley, M (2004) Nurse leadership within primary nursing care: the perceptions of community nurses, GPs, policy makers and members of the public. J Nurs Manag 12: 69-76.

Narinen, A, Kekki, P (2003) The content of the nurse manager's work in Finland. Vard Nord Utveckl Forsk 67 16-20.

Pearson, A, Laschinger, H, Porritt, K, Jordan, Z, Tucker, D, Long, L (2007) Comprehensive review of evidence on developing and sustaining nursing leadership that fosters a healthy work environment in healthnursing care. I J Evid Based Health. Nurs care 5: 208-253.
Reinhardt, AC (2004) Discourse on the transformational manager metanarrative or finding the right person for the job. Adv Nurs Sci 27: 21-31.

Sellgren, S, Ekvall, G, Tomson, G (2006) Leadership styles in nursing management: preferred and perceived. J Nurs Manag 14: 348-355.

Stanley, D (2006a) In command of nursing care: clinical leadership explored. J Res Nurs 11: 20-39.

Stanley, D (2006b) In command of nursing care: toward the theory of congruent leadership. J Res Nurs 11: 132144.

Upenieks, V (2003) What constitutes effective leadership? Perceptions of magnet and non-magnet nurse managers. J Nurs Adm 32: 456-467.

Viitanen, E, Wiili-Peltola, E, Tampsi-Jarvala, T, Lehto, J (2007) First-line nurse managers in university hospitalscaptives to their own professional culture. J Nurs Manag 15: 114-122.

Wieck, KL, Prydun, M, Walsh, T (2002) What the emerging workforce wants in its managers. J Nurs Sch 34: 283-288.

Terese Bondas ( $\mathrm{PhD}$, LicNsc, MNsc, RN) is a Professor at the University College of Borås, Department of Caring Science, Sweden; Associate Professor, Kuopio University, Department of Nursing Science, Finland; and Chairperson of BfiN-Childbearing in the Nordic Countries - the qualitative research network (http://www.bfin.hb.se) and NiV-the Nordic Research Network in Leadership in Health Care. Terese Bondas has research interests in Leadership in evidence-based care and childbearing and metasynthesis and qualitative research.

Email: terese.bondas@hb.se 